[Aus dem Königl. Institut für Infektionskrankheiten zu Berlin.] (Direktor: Geh. Obermed.-Rat Prof. Dr. Gaffk y.)

\title{
Über die Bedeutung und Tätigkeit des großen Netzes bei der peritonealen Infektion.
}

\author{
Von
}

\author{
Prof. Dr. Josef Koch, \\ Mitglied des Instituts. \\ (Hierza Taf. II u. IIL.)
}

Das Schicksal der in die Bauchböhle von Versuchstieren injizierten Baliterien ist schon häufig Gegenstand der Untersuchung gewesen, hauptsächlich aus dem Grunde, weil die dabei im Peritonealraum sich abspielenden Vorgänge besonders geeignet sind, über prinzipielle Fragen der Bakteriologie, nämlich über die wichtige Frage des Unterganges der Bakterien und die dabei gebildeten bakteriziden Substanzen Klarheit zu schaffen.

Während bekanntlich R. Pfeiffer die Ansicht rertritt, daB durch die bei der Immunisierung gebildeten Bakteriolysine der freien Peritonealflüssigkeit die Auflösung der pathogenen Keime herbeigeführt wird, sind es nach Metschnikoff zelluläre Vorgänge, besonders die aktive Phagozytose, durch welche die ins Peritoneum gebrachten Bakterien abgetötet werden.

Trotz zahlreicher einschlägiger Arbeiten ist eine Einigung dieser beiden Anschauungen bisher nicht exzielt worden.

Um mir ein eigenes Urteil zu bilden, habe ich eine Reihe von Untersuchungen angestellt und zunächst die Frage zu beantworten gesucht, was aus verschiedenen in die Bauchhöhle der Versuchstiere gebrachten Bakterien wird. Im Verlauf dieser Arbeit bin ich zu der Ansicht gekommen, daB 
zwar Bakteriolyse und Phagozytose außerordentlich wichtige Mittel des Organismus im Kampfe mit der Infektion darstellen, daB aber damit die Zahl der mitwirkenden Faktoren noch nicht erschöpft ist; keineswegs dürfen wir uns die Bauchhöhle als einen Raum vorstellen, in dem nur der Lymphe und den Leukozyten die Zerstörung der eingedrungenen Organismen obliegt, vielmehr ist der Ablauf der Vorgänge wesentlich komplizierter. Vor allen Dingen spielt bei diesen Vorgängen ein Organ eine auBerordentlich wichtige Rolle, dessen Bedeutung und Tätigkeit bisher kaum erkannt ist, nämlich das grobe Netz.

Bevor ich zum Studium des Verhaltens der Bakterien im Bauchraum überging, habe ich eine Reihe von Vorversuchen mit körnchenförmigen Substanzen angestellt, die ich in die Peritonealhöhle der Versuchstiere einspritzte. Es ist bereits bekannt, dab sehr fein verteilte Substanzen wie z. B. Tusche- oder Zinnoberemulsionen bei Einführung in die Körpergewebe sich zunächst ebenso verhalten wie Bakterien. Man hat sich daher schon vielfach in der experimentellen Pathologie derartiger Substanzen mit großem Nutzen bedient, um die Wege kennen zu lernen, die pathogene Keime im lebenden Organismus einschlagen. Die Anwendung feinster farbiger korpuskulärer Elemente hat außerdem noch den Vorzug, daß sie durch ihre Farbe sich leichter vom Körpergewebe abheben und auf diese Weise später leichter nachgewiesen werden können.

Wie ich mich durch sehr zahlreiche Versuche überzeugt habe, besteht in der Tat eine sehr weitgehende Utbereinstimmung des Verhaltens fein verriebener Tuscheemulsion mit Bakterienaufschwemmungen, soweit die spezifische Wirkung des betreffenden Bakteriums zunāchst auBer Betracht bleibt, die für jeden Mikroorganismus besonders festgestellt werden muB. Hiervon abgesehen, ist aber die Anwendung von fein verteilten Tuscheemulsionen ein ausgezeichnetes Hilfsmittel und ein vorzūglicher Wegweiser beim Studium und für die Beurteilung der späteren Versuche mit Bakterien.

\section{I. Über das Schicksal der in die Bauchhöhle der Versuchstiere injizierten Tusche.}

Über die Technik bemerke ich folgendes: Zur Injektion benutzte jeh feinste chinesische Tusche, die auf einem Reibbrett möglichst fein in Kochsalzlösung verrieben wird und je nach Belieben mehr oder weniger konzentriert hergestellt werden kann. Als Versuchstiere dienten mir Mäuse, Ratten, Meerschweinchen, Kaninchen, Hunde, Affen, denen wechselnde Mengen in die Bauchhöhle eingespritzt wurden. Bei Mäusen genügen 0.2 , Meerschweinchen 1.0, Ratten, $0 \cdot 5$, Kaninchen $2 \cdot 0^{\mathrm{cem}}$ einer dichten Tuscheaufschwemmung. Es empfiehlt sich, zunächst mit den kleinen Versuchstieren zu beginnen und dann die Versuche an größeren Tieren vorzunehmen. Das ideale 
Tier für derartige Versuche ist das Meerschweinchen, auf das sich die folgenden Angaben zunächst beziehen.

Spritzt man einem Meerschweinchen von etwa $250 \mathrm{grm} 1 \mathrm{ccm}$ Tuscheemulsion ein und entnimmt nach etwa einer Stunde mittelst feiner Glaskapillaren Peritonealflüssigkeit, so kann man zunächst konstatieren, daB die Flüssigkeit im Bauchraum stark vermehrt ist. Das geht schon daraus hervor, daß sie bei der Entnahme förmlich in die Kapillare hineinschieBt. Durch die Beimischung der Tusche ist das Transsudat intensir schwarz gefärbt. Im hängenden Tropfen sieht man wenige Leukozyten und die Tusche als unregelmäBige, in lebhafter Molekularbewegung befindliche schwarze Teilchen. Nach 2 Stunden ist die Zahl der Leukozyten bedeutend vermehrt, und man bemerkt, daB die meisten bereits Tuscheteilchen ihrem Protoplasma einverleibt haben. Die Farbe des Transsudates ist dabei schon wesentlich heller geworden. In den folgenden Stunden nimmt seine Mlenge bedeutend ab, die Farbe wird heller, die Leukozyten beladen sich mehr und mebr mit der Tusche und nach 24 Stunden ist gewöhnlich diese aus der freien Peritonealflüssigkeit, die wieder ihre klare Farbe angenommen hat, verschwunden. Die Tuscheteilchen befinden sich jetzt innerhalb der Leukozyten, vorausgesetzt, daB nicht eine zu grobe Menge von Farbe einverleibt wurde. Es hat also eine außerordentlich starke Phagozytose stattgefunden, die im allgemeinen mit der Menge der eingeführten Tusche parallel läuft. Das Bild, das ein nach 24 Stunden hergestellter hängender Tropfen der Peritoneallymphe darbietet, ist ein höchst eigenartiges. Die Tuscheteilchen sind von den Granula der Lenkozyten adsorbiert. Weitere Einzelheiten übergehe ich hier und verweise auf meine Publikation. ${ }^{1}$

Wird das Versuchstier nach 24 Stunden getötet, so kann man folgenden anatomischen Befund erheben. Die freie Peritoneallüssigkeit ist kaum vermehrt, die Menge entspricht ungefähr der eines normalen Tieres. Einen eigentümlichen Anblick gewährt das Netz, das gewöhnlich aufgerollt unterhalb des Magens liegt. Es sieht intensiv schwarz aus, als wenn sämtliche Tusche sich anf ihm niedergeschlagen hätte. Breitet man es aber auf einer Glasplatte vorsichtig aus, so sieht man, daB die Tusche nicht auf dem Netz, sondern innerhalb der zarten Gewebsmaschen in zierlicher Weise gleich einem Adergeflecht angeordnet sich befindet. Schwärzliche Streifen begleiten meist die vom Fettgewebe begleiteten Gefäße. Dabei treten einzelne Fleckchen und Knötchen von miliarer bis LinsengröBe durch ihre intensiv schwarze Färbung besonders stark hervor,

\footnotetext{
${ }^{1}$ Josef Koch, Über den Mechanismus der Phagozytose. Diese Zeitschrift. 1911. Bd. LXVIII. S. 80 . 
als wenn die Tusche sich hier noch stärker angehäuft hätte. Gewöhnlich liegen auBerdem noch einzelne schwärzliche Massen, die aus einer Unzahl von Leukozyten mit phagozytierter Tusche bestehen, zwischen den Darmschlingen oder in den Buchten der Peritonealhöhle des Versuchstieres. Sie lassen sich von der Serosa leicht abheben, während dies beim Netz nicht der Fall ist.

So oft man den Versuch bei den verschiedenen Versuchstieren, Maus, Ratte, Meerschweinchen, Kaninchen, Hund auch wiederholen mag, immer ist es derselbe Befund, stets findet sich fast die gleiche Menge der Tusche im Gewebe des Netzes.

In derselben Weise fiel auch ein Versuch bei einem Affen aus, der $7^{\mathrm{ccm}}$ einer Tuscheemulsion intraperitoneal erhalten hatte; doch war hier die Tusche, als das Tier nach 24 Stunden getötet wurde, über das sehr faltenreiche groBe Netz viel gleichmäBiger verteilt. Nur die fettgewebsreichsten Partien zeigten eine intensivere und diffusere Färbung; stärkere knötchenförmige Anhäufungen der Tusche wie bei den kleineren Tieren habe ich hier nicht beobachtet, dagegen zeigte das Centrum tendineum des Zwerehfells eine dichte schwarze Streifung, während das Peritoneum parietale und die Serosa der Därme keine Veränderungen zeigten.

Das Netz hat also die Fähigkeit, fein verteilte körnchenförmige Substanzen nach Injektion in die Bauchhöhle zu resorbieren. AuBer dieser starken und regelmäBigen Resorption durch das Netz ist gewöhnlich eine weit geringere durch die Lymphbahnen des Centrum tendineum des Zwerchfells zu konstatieren, das bei diesen Versuchen eine mehr oder minder starke Strichelung in seiner Kuppe aufweist. Man kann dies besonders dann beobachten, wenn gröBere Mengen der Farbstoffemulsion eingespritzt werden. Auch hier liegt die Tusche im Gewebe, wie man sich leicht an Schnittpräparaten überzeugen kann; auch läBt sie sich durch Abwischen nicht entfernen. DaB die Lymphbahnen des Zwerchfells sich an der Aufsaugung peritonitischer Exsudate beteiligen, ist übrigens eine bekannte Tatsache.

Auffällig ist jedoch das Verhalten der übrigen Peritonealböhle, des Peritoneum parietale und der Serosa der Darmschlingen, die sich an der Resorption körnchenförmiger Substanzen nicht beteiligen. Jedenfalls babe ich im Tuscheversuch nichts gefunden, was auf eine nennenswerte Resorption korpuskulärer Substanzen seitens dieser Teile des Peritoneums schließen läßt. Für die mangelnde Resorptionsfähigkeit dieser Teile spricht auch der Umstand, daß sowohl Phagozyten, die sich mit Tusche beladen haben, als auch Haufen von verklumpten Phagozyten längere Zeit im Peritonealraum unverändert liegen bleiben. 
Aus den Versuchen geht demnach hervor, daB die Entfernung korpuskulärer Elemente aus der Bauchhöhle in erster Linie und weitaus zum gröBten Teile durch das groBe Netz, in $\mathrm{z}$ weiter Linie durch die Lymphbahnen des Centrum tendineum des Zwerchfells bewirkt wird.

Auf das Verhalten des groBen Netzes gegenüber den in die Bauchhöhle gebrachten körnchenförmigen Substanzen haben bereits Heusner ${ }^{1}$, Heger ${ }^{2}$, Rose ${ }^{3}$ aufmerksam gemacht. Heusner hat sich damit begnügt, lediglich die Tatsache zu konstatieren, ohne auf den wichtigen und interessanten Mechanismus der Resorption seitens dieses Organes näher einzugehen.

Es sei mir gestattet, an dieser Stelle zunächst etwas Näheres über die Anatomie und die herrschenden Ansichten über die F'unktion des Omentum majus vorauszuschicken:

Heusner leitet seine Arbeit „Die physiologisehe Bedeutung des groBen Netzes" mit den Worten ein: „Die Bestimmung des groBen Netzes der Säugetiere ist eine ungelöste Frage, und der Streit darüber reicht bis ins Altertum zurück." Und nach einem Rückblick faßt er die zurzeit bestehenden Ansichten also zusammen: „Neue Autoren sehen in der Netzschürze ein Absonderungs- und Befeuchtungsorgan oder auch ein Resorptionsorgan, wieder andere einen Blut- und Lymphbereitungsapparat. In der neuesten Zeit ist das groBe Netz infolge der Wichtigkeit, welches es für die Chirurgie erlangt hat, von verschiedenen Seiten zu einer Schutzvorrichtung gegen pathologische Zufälle erhoben worden, und AlbrechtFrankfurt vergleicht seine Tätigkeit mit jener der Leukozyten, deren Fähigkeiten ebenfalls bei Entzündungsprozessen zur vollen Entfaltung gelangen können." Nach Heusner kann jedoch das große Netz seiner Hauptbestimmung nach nicht als eine Schutzvorrichtung bezeichnet werden; denn es gibt kein Organ für pathologische Ausnahmefälle.

Ober die Anatomie des Netzes, vor allem über die Frage, ob hier Lymphgefäße vorkommen oder nicht, sind die Ansichten geteilt. Nach Suzuki ${ }^{4}$ sind bezüglich der Art der Resorption im Laufe der Zeit zwei vollkommen divergierende Ansichten hervorgetreten; während die einen

${ }^{1}$ Heusner, Die physiologische Bedeutung des groBen Netzes. Münchener med. Wochenschrift. 1905.

${ }^{2}$ Heger, Contribution à l'étude expérimentale des fonctions du grand épiploon. Annales de la Soc. Royale des sciences médicales de Bruxelles. 1904. T. XIII.

${ }^{3}$ Rose, Das Verhalten des groBen Netzes nach intraperitonealen Injektionen körniger Stoffe. Inaug.-Diss. StraBburg 1907.

4. Suzuki, Über die Resorption im Omentum majus des Menschen. Virchows Archiv. Bd. CCII. 
Autoren, z. B. Albert von Haller, Chaussier und Adelon, Eccles, Norris, Luschka, resorbierende LymphgefäBe im groBen Netz beschrieben haben, leugnen andere ihr Vorkommen im groBen Netz. In den meisten Lehrbüchern der normalen Anatomie findet sich nichts näheres über die LymphgefäBe des Omentum majus erwähnt, selbst nicht in dem ausgezeichneten Werke von Bartels" „Das Lymphgefäßsystem". Auf die Literatur näher einzugehen, $m a B$ ich mir hier versagen; ich verweise auf die literarische Übersicht bei Suzuki.

Suzuki selbst hat auf Anregung Chiaris, der in einem Falle von in Spontanheilung begriffener lokaler AbreiBung des linken Leberlappens in Schnitten vom großen Netz Leberzellen und Gallengangsepithelien in erweiterten Lymphgefäßen gefunden hatte, die Frage der Lymphgefäße und den Nachweis resorbierter Substanzen beim menschlichen Netz an einem gröBeren Material studiert. Durch die mikroskopische Untersuchung konnte er in den Lymphgefäßen sehr verschiedenen Inhalt nachweisen, Leberzellen, Gallengangsepithelien, Pankreaszellen, Geschwulstzellen, rote und weiße Blutkörperchen und auch Bakterien. Alle diese Elemente waren, wie Suzuki schreibt, zweifellos von der Bauchhöhle in die LymphgefāBe des Omentum majus gelangt. Er zieht aus seinen Untersuchungen den SchluB, daB 1 . das Omentum majus regelmäBig reichliche Lymphgefäße besitzt, daB 2. die verschiedenen korpuskulären Elemente, die in der Bauchhöhle als abnormer Inhalt auftreten, auch in die Lymphgefäße des Omentum majus eintreten, und daß 3. infolgedessen das Omentum majus bei Infektionen des Peritoneums als ein schützendes Organ der Bauchböhle anzusehen ist, ja, daß es geradezu sehr wesentlich daran betejligt ist, die Bauchhöhle rein zu halten.

Auch nach meinen Untersuchungen ist das Netz ein Organ, das neben Blutgefäßen regelmäßig Lymphgefäße enthālt. Nicht nur beim Menschen, sondern auch bei den kleineren Säugetieren gelingt es, nach zahlreichen eigenen Untersuchungen in Netzschnitten Lymphgefäße und Lymphknoten nachzuweisen. Norris ${ }^{2}$ hat die Verhältnisse beim Kaninchen genauer untersucht. Er teilt die LymphgefäBe in zwei Gruppen ein; die größeren tiefen verlaufen in Begleitung der Blutgefäße, während die kleinen oberflächlichen nach allen Richtungen das Netz durchziehen. Den Anfang der kleinen Lymphgefäße sollen die sogenannten von Recklinghausenschen Saftkanälehen bilden. Diese kommunizieren mit den Lymphkapillaren und diese münden in die groBen Lymphstämme. Die Lymph-

\footnotetext{
${ }^{1}$ Bartels, Das LymphgefäBsystem. 17. Lieferung des Handbuches der Anatomie des Menschen. Herausgegeben von Karl von Bardeleben. Bd. III. Abt. IV.

${ }^{2}$ Norris, Bull. med. Univ. of Pennsylv. 1908-19u9. Vol. XXI.
} 
Über die Bedeutung v. Tätigkett des grossen Netzes Usw. 423

gefäße des großen Netzes laufen in mehreren Lymphdrüsen im obersten Teil des groBen Netzes an der Curvatura major ventriculi zusammen.

Uber die sonstige feinere Histologie des großen Netzes sei folgendes bemerkt: Beim Mensehen zeigt es areolären Bau und besteht aus anastomosierenden Bindegewebsbündeln, die ein zierliches Netz mit polygonalen oder rechteckigen Maschen bilden und Fibroblasten enthaiten. Die Lymphgefäße verlaufen nach Suzuki meist mit den Blutgefäßen, zuweilen aber auch zwischen den Blutgefäßen. Von Recklinghausen hat zur Unterseheidung der Lymph- und BlutgefäBe, die sehr schwierig ist, angegeben, daB die Wandungen der LymphgefäBe an den Knotenpunkten nach auBen hin konkar sind.

Ein Stück Netz eines jungen normalen Kaninchens vom proximalen, unterhalb des Magens gelegenen Teil habe ich auf der Taf. II, Zeichnung Nr. 1 wiedergegeben. Wie die Abbildung zeigt, kommen hier (auch beim Meersehweinchen) 1. teils diffuse, teils schärfer begrenzte, an Lymphfollikel erinnernde Zellkomplexe verschiedener Größe vor, die bis auf die gröBten durchweg einer bindegewebigen Umhüllung entbehren. Im ganzen Netz, besonders aber auch in der Nähe dieser aus Lymphozyten bestehenden Zellaggregate sieht man 2. zahlreiche große und polymorphkernige Zellen (s. Taf. II, Fig. 2), deren Protoplasma aus zahlreichen eosinophilen Granula besteht. In sehr groBer Anzahl habe ich sie auch entlang den Blut- und LymphgefäBen, dem Fettgewebe, das die GefäBe einscheidet, angelagert gefunden. Es sind starke Phagozyten. Im Tuscheversuch, der über die Verbreitung dieser Zellen im Netz sehr guten AufsehluB gibt, kann man sehen, dab ihre Granula die Farbstoffteilehen massenhaft adsorbiert haben.

Uber die zelligen Eliemente der Lymphe und der serösen Höhlen, sowie über die physiologische Bedeutung des Netzes haben Weidenreich ${ }^{1}$ und Schott ${ }^{2}$ wertrolle Mitteilungen gemacht.

Nach Weidenreich enthalten die serösen Höhlen, besonders die Bauchhöhle, in ihrem spärlichen normalen Transsudat stets Zellelemente. Bei allen Tieren kommen große protoplasmareiche rundkernige Zellen vor, die als abgestoBene Endothelien oder eingewanderte Blutelemente (Lymphozyten) oder als losgelöste adventitielle Zellen der NetzgefāBe angesprochen

\footnotetext{
1 Weidenreich, Über die zelligen Elemente der Lyymphe und der serösen Höhlen. Verhandl. der Anatom. Gesellschaft auf der 21. Versammlung am 24. bis 27. April 1907 zu Würzburg.

2 Schott, Morphologische und experimentelle Untersuchungen über Bedeutung und Herkunft der Zellen der serösen Höhlen und der sogenannten Makrophagen. Inaug.-Diss. Strabburg. Archiv für mikr. Anatomie und Entwicklungsgeschichte. 1909. Bd. IXXIIV.
} 
werden. Daneben finden sich z. B. beim Meerschweinchen zahlreiche rund- und polymorphkernige eosinophile Leukozyten und spärliche Lymphozyten. Sehr spärlich sind die polynukleären Leukozyten vertreten.

Trifft das Peritoneum ein Reiz, so ändert sich das Bild, indem sehr zahlreiche polynukleäre Leukozyten aus den hyperämischen GefäBen austreten. Diese Leukozyten gehen nach Weidenreich im Exsudat sehr schnell zugrunde. Entweder zerfallen sie, oder sie werden von den großen rundkernigen Zellen (Makrophagen) aufgenommen. Bei wiederholter Reizung wandern aus dem Blut keine polynukleären Leukozyten mehr aus. Das Ezsudat besteht fast nur noch aus Mrakrophagen, aus jenen groben rundkernigen Zellen und eosinophilen rund- und polymorphkernigen Leukozyten. Die beiden Formen zeigen nach Weidenreich zahlreiche Mitosen. Es handelt sich also hier um eine autochthone Zellbildung innerhalb der Bauchböhle. Weidenreich behauptet, daß die groBen protoplasmareichen rundkernigen Zellen, die nach ihm charakteristische Bestandteile der normalen und der entzündlichen Peritonealflüssigkeit darstellen, losgelöste Peritonealendothelien sind, die ihre phagozytäre Eigenschaft in hohem MaBe auch innerhalb des Netzes entfalten und die aus dem Blut austretenden polynukleären Leukozyten sofort in sich aufnehmen.

Nach meinen Untersuchungen kommen als phagozytäre Elemente der Bauchhöhle zwei große Zellengruppen in Betracht, die wir mit Metschnikoff als Makro- und Mikrophagen bezeichnen lönnen.

Die Mikrophagen oder polynukleären Leukozyten stammen aus dem Blut und wandern, sobald die Peritonealhöhle von einem Reiz betroffen wird, aus den hyperämischen GefäBen des Netzes aus.

$\mathrm{Zu}$ den Makrophagen rechne ich die großen mononukleären, protoplasmareichen rundkernigen Zellen, die hauptsächlich minderwertige und abgestorbene Zellelemente aufnehmen, ror allem aber die eosinophilen Zellen, deren Protoplasma aus einer Unzahl von Granula besteht. Diese beiden verschiedenen Makrophagen stammen nicht aus dem Blut, sondern aus dem Netz; die auf der Taf. II, Figur Nr. 2 abgebildeten Zellen sind keineswegs identisch mit den eosinophilen Leukozyten des Blutes. Bei bakterieller peritonealer Infektion sind es diese Makrophagen hauptsäehlich, die Mikroorganismen sowie Furbstoffteilchen vermittelst ihrer Granula in großer Menge adsorbieren und in ihrer FreBtätigkeit den polynukleären bei weitem überlegen sind.

Auf Grund meiner Untersuchungen stimme ich mit Weidenreich und Schott darin überein, daB die Makrophagen des entzündlichen Peritonealexsudates ursprünglich sessile Zellen des Netzes sind, die auf einen entzündlichen Reiz zu 
đber die Bedeutung U. Tätrakeit des grossen Netzes USW. 425

Phagozyten werden, indem sie sich zum Teil aus dem Zellverbande lösen und zu freien Elementen werden.

Auch über die physiologische Bedeutung des Netzes bin ich zu derselben Ansicht wie Weidenreich gekommen, der folgende Auffassung vertritt.

„Das Netz ist zu charakterisieren als ein in der Fläche entfalteter lymphoider Apparat, gleichwertig dem Reticulum der Lymphbahnen und der Sekundärknötchen der Lymphknoten nebst den in ihnen enthaltenen Zellen. Dieser lymphoide Apparat ist aufgehängt in der einem Lymphraum entsprechenden Peritonealhöhle; das Netz ist durchbrochen, so daB Flüssigkeit wie korpuskuläre Elemente hindurchpassieren können, wie die Lymphe durch das Reticulum der Lymphbahnen. Allenthalben im Netz können Sekundärknötchen (Ansammlung von Lymphozyten) sich bilden, die sogenannten "tĥtuhes laiteuses" Ranviers. Physiologisch wirken die Zellen des Netzes wie die der Lymphdrüsen, indem sie sowohl Zellen produzieren, die frei werden können und in den Lymphraum gelangen das sind jene großen rundkernigen Zellen der Peritonealflüssigkeit, als auch solche, die innerhalb des Organs selbst Verwendung finden - dieselben Zellen und Lymphozyten."

Auf welche Weise kommt nun die Aufnahme körnchenförmiger Substanzen durch das Netz zustande?

Daß nach der Einspritzung frei im Exsudat der Bauchböhle schwimmende Tuscheteilchen nach 24 Stunden in erheblicher Menge von den Leukozyten phagozytiert sind, steht fest. Das Fortschreiten der Phagozytose läBt sich ja durch die Kontrolle des Peritonealexsudates im Laufe der 24 Stunden genau verfolgen. Die Menge der nach 24 Stunden innerhalb der Bauchböhle in den Phagozyten gelegenen, von den Granula adsorbierten Tuscheteilchen entspricht aber nicht im entferntesten der ganzen eingespritzten Menge, sondern sie stellt nur einen kleinen Teil, gewissermaBen nur den Rest der im Bauchraum zurückgebliebenen Farbstoffteilchen dar. Die Hauptmenge ist aus ihm verschwunden, und da man bei der Ontersuchung des Netzes - die sehr leicht auszuführen ist, indem man die durchsichtigen Partien über einem Objektträger ausbreitet und unter dem Mikroskop betrachtet - konstatieren kann, daB die Tuscheteilchen sich in einer Unmenge von Phagozyten innerhalb des Netzes eingeschlossen finden, so müssen wir annehmen, daB die Phagozyten in den Peritonealraum eingewandert, die Farbteilchen phagozytiert und ins Netz zurückgewandert sind. Diese vermittelst der Phagozyten stattfindende Resorption korpuskulärer Elemente aus der Bauchhöhle in das groBe Netz möchte ich als indirekte bezeichnen. 
Bei der Untersuchung des Netzes derartiger Versuchstiere sieht man jedoch, daB nicht nur die eigentlichen Wanderzellen, die Mikro- und Makrophagen, sondern auch fixe Phagozyten, nämlich die Endothelien der LymphgefäBe, die Farbstoffteilchen aufgenommen haben. Ja man findet die Tusche hier und da auch frei im Lumen der LymphgefäBe. Daraus geht mit Sicherheit hervor, daB die LymphgefäBe des Netzes korpuskuläre Elemente auch direkt ohne Vermittlung der Phagozyten aufsaugen können. Es kommt also neben der indirekten auch eine direkte Resorption durch die Lymphbahnen des Netzes vor. Hierfür spricht auch die Schnelligkeit der Aufnahme; denn schon kurze Zeit nach der Injektion liegt ein großer Teil des Farbstoffes im Netz, wovon man sich leicht nach Tötung des Tieres überzeugen kann, während Leukozyten im allgemeinen in den ersten 2 Stunden nach der Einspritzung nur spärlich im Peritoneum vorhanden sind.

Die mit den Farbstoffteilchen beladenen Makrophagen trifft man teils einzeln, teils zu Gruppen vereinigt, in den durchsichtigen Partien des Netzes an; vor allem aber findet man sie in groBer Anzahl zu beiden Seiten des Fettgewebes, in das die GefäBe eingebettet sind, so daB schon bei makroskopischer Betrachtung diese Stellen als schwarze Streifen auffallen. In die GefäBe selbst dringen sie nicht ein. Ich habe bei zahlreichen Untersuchungen kein einziges Gefäß getroffen, wo ein Tuscheleukozyt in einem GefäB, Vene oder Arterie, gelegen hätte.

Eine starke Anhäufung der Tuschephagozyten findet weiter in den Lymphknötchen, und besonders in den an der Wurzel des Netzes gelegenen Lymphknoten statt. Diese fallen daher schon makroskopisch durch ihre intensiv schwarze Farbe auf. Um ein klares Bild der Verhältnisse zu gewinnen, ist es nötig, diese Partien am gehärteten Präparat zu studieren. Man kann dann sehen, dab die Aufnahme der Tusche seitens der Phagozyten innerhalb der Lymphknötehen eine durchaus ungleichmäBige ist. Die Mikrophagen enthalten zwar fast ohne Ausnahme kleine Tuschepartikelchen in ihrem Zellenleibe, doch werden sie von anderen Zellen ganz in den Schatten gestellt, die vollgepfropft mit Tusche, wie schwarze Kugeln vom übrigen Gewebe sich abheben. Diese Zellen sind die zwei verschiedenen Arten der Makrophagen. Sie entstammen, wie bereits oben bemerkt, nicht dem Blute, wie die polynukleären Leukozyten, sondern dem lymphoiden Apparate des groben Netzes.

Von hohem Interesse und für den Mechanismus der Entstehung einer allgemeinen Infektion vom Peritoneum aus wichtig ist die Frage nach dem weiteren Schicksal der körnchenförmigen Substanzen. Ich hatte bereits festgestellt, daB der bei weitem gröBte Teil der in die Bauchhöhle injizierten Farbstoffmenge von den leukozytären Elementen des Netzes 
und den . Lymphknötchen festgehalten wird, und daB ein Rest innerhalb der Leukozyten in der Bauchhöhle selbst liegen bleibt. Weiter hatte ich mitgeteilt, daß ein geringer Teil durch die Lymphbahnen des Centrum tendineum aufgenommen wird. Diese Lymphbahnen perforieren das Zwerchfell und nehmen ihren weiteren Verlauf mit den Venae mediastinales retrosternal, wo sie oberhalb der Brustapertur ihren Inhalt in den großen Ductus chyliferus abführen. In dem Verlauf dieser retrosternalen Lymphstränge sind Lymphknoten eingeschaltet, und zwar liegen beim Meerschweinchen und Kaninchen je zwei ungefähr in der Höhe der dritten Rippe, zwei oberhalb der oberen Brustapertur. Auch beim Affen und beim Mensehen finden sich im Verlauf der restrosternalen LymphgefäBe kleine Lymphdrüsen. Wenn man den Versuchstieren reichlich Tusche intraperitoneal injiziert und die Tiere nach 48 Stunden tötet, so heben sich diese Lymphknötchen durch ihre intensiv schwarze Farbe deutlich von ihrer Umgebung ab, ein Beweis, daB die Farbstoffteilchen hier abgelagert wurden. Die Kenntnis dieser, der Peritonealhöhle entstammenden, das Zwerchfell perforierenden Lymphbahnen mit ihren eingeschalteten Lymphknötehen ist von größter Wichtigkeit; denn sie zeigen uns den Weg, auf dem ein Bruchteil der korpusliulären Substanzen und Bakterien in die Lymphe und sekundär in den allgemeinen Blutkreislauf geraten kann. Die Infektion des Blutes durch Bakterien vom infizierten Peritoneum aus erfolgt also nicht direkt durch die BlutgefäBe der Peritonealhöhle, sondern auf indirektem Wege durch die ableitenden LymphgefäBe. Aber auch hier hat der zweckmäßig gebaute Organismus gegen eine ungehinderte Überflutung des Blutes Barrieren geschaffen, indem in die ableitenden Lymphbahnen Lymphknoten eingeschoben sind, die den größten Teil der mit fremden Bestandteilen beladenen Leukozyten abfangen.

Für die von mir geschilderte Infektion des Blutes und weiterhin der verschiedenen Körperorgane von der Peritonealhöhle aus lassen sich noch weitere Beweise anführen. Wenn man Tuschetiere nach 24 Stunden tötet und vom Parenchym der Leber und Milz Abstriche macht, so findet man fast stets spärliche Tuschezellen, und zwar die großen mononukleären Phagozyten. Da weder Leber noch Milz direkte Verbindungen mit dem freien Peritonealraume haben, so können sie nur auf dem eben geschilderten Wege mit dem Blutstrom in diese Organe gelangt sein. Wenn wirklich eine direkte Kommunikation dieser Organe mit der Bauchhöhle bestände, so müBten die Tuschephagozyten weit zahlreicher hier angetroffen werden; aber nur wenigen Zellen gelingt es, alle Barrieren zu passieren, ehe sie in den Hauptlymphstrom und sekundär in die allgemeine Blutzirkulation gelangen. 


\section{II. Über das Schicksal der in die Peritonealhöhle injizierten Bakterien.}

Nachdem vermittelst des Tuscheversuches das Schicksal der in die Bauchböhle injizierten Farbstoffemulsionen festgestellt war, war es nicht schwer, dasjenige der Bakterien genauer zu verfolgen. Die Frage, auf welche Weise Bakterien aus der Bauchhöhle verschwinden, hat seit Beginn der balteriologischen Ära die Forscher gefesselt, und besonders in letzter Zeit ist das Problem ron chirurgischer Seite verschiedentlich experimentell zu lösen versucht worden.

Peiser ${ }^{1}$ und Glimm ${ }^{2}$ sind der Ansicht, daß die Bakterien von den Iyymphbahnen des Peritoneums aufgenommen werden; indes liegen, wie Danielsen ${ }^{3}$ bemerkt, bisher keinerlei sichere Beweise für diese Behauptung vor. Nach ihm muB auch die Vermutung anderer Forscher (Bertelsmann), die Bakterien würden durch die Blutbahn aus der Bauchhöhle resorbiert, so lange respektiert werden, bis vollgültige Beweise vorliegen.

Danielsen selbst hat die Frage durch Tierversuche zu entscheiden versucht. Zu diesem Zweck legte er bei Hunden den Ductus thoracicus frei und versuchte, in ihn eine feine Kanüle einzubinden, oder begnügte sich mit der Freilegung und entnahm dem eröffneten GefäB Lymphe mit der ausgeglühten Platinschlinge. In die freigelegte Carotis externa band er eine ausgekochte Glaskanüle ein, darauf injizierte er eine ganze, in steriler Kochsalzlösung aufgeschwemmte Colikultur in die Bauchhöhle; in verschiedenen Zwischenräumen wurde dann Blut und Lymphe entnommen und auf passende Nährböden übertragen.

Bei den in dieser Weise angestellten Versuchen konnten in der Lymphe des Ductus thoracicus die in die Bauchhöhle gebrachten Colibakterien nachgewiesen werden, während in dem zu gleicher Zeit entnommenen Blut sich kein einziges Bacterium coli zeigte.

Da es mir darauf ankam, zunächst eimmal den reinen Resorptionsvorgang eines Bakteriums, das sich der bakteriziden Tätigkeit der freien Peritonealflüssigkeit gegenüber resistent erwies, kennen zu lernen, so habe ich meine Versuche mit Tuberkelbazillen menschlichen und tierischen Ursprungs begonnen.

Die Kaninehen von $1500 \mathrm{grm}$ Gewicht erhielten $0.03 \mathrm{grm}$ (Typus bovinus), Meerschweinchen $0.02 \mathrm{grm}$ (Typus humanus) einer feinen, in Kochsalzlösung verriebenen Aufschwemmung von Tuberkelbazillen intra-

'Peiser, Bruns Beiträge. Bu. XI,V.

${ }^{2}$ Glimm, Deutsche Zeitschrift für Chirurgie. Bd. IXXIII.

${ }^{3}$ Danielsen, Über die Schutzvorrichtungen in der Bauchhöhle. Habilitationsschrift. Laul' l jr., Tübingen 1907. 
peritoneal eingespritzt. Zwar ist die Menge der injizierten Bazillen immerhin eine beträchtliche; aber für den Zweck der Feststellung der Resorption und des ersten Verlaufes der Infektion war eine größere Dosis vorzuziehen, da die Bazillen im infizierten Tiere dann später leichter aufgefunden werden konnten.

Entnimmt man 1 Stunde nach. der Einspritzung Peritonealexsudat, so ist die Phagozytose bereits im Gange, aber es sind doch nur verhältnismäBig wenige Mikro- und Makrophagen sichtbar. In den folgenden Stunden nimmt die Leukozytose $\mathrm{zu}$, und man sieht schon zahlreiche Stäbchen im Innern der Phagozyten.

Nach 7 Stunden ist das Peritonealexsudat so reich an poly- und mononukleären Leukozyten, daB man glaubt, Eiter vor sich zu haben. Etwa ein Drittel der Zellen haben Tuberkelbazillen im Innern.

Die mononukleären zeichnen sich dadurch aus, daB sie meist vollgestopft sind mit Bazillen, während die polynukleären meist nur vereinzelte Stäbchen aufgenommen haben. Freie Bazillen findet man in der Peritonealflüssigkeit nur mehr ganz vereinzelt.

Nach 9 Stunden hat sich das Bild insofern geändert, als etwa $2 / 3$ der Zellen Tuberkelbazillen phagozytiert haben. Außerhalb der Zellen befindet sich kaum noch ein Bacillus.

Nach 24 Stunden enthālt die Bauchhöhle noch zahlreiche Phagozyten, doch hat die Zahl der Zellen, die Bazillen in ihrem Protoplasma aufweisen, stark abgenommen.

Will man sich Klarheit darüber verschaffen, wo die Bazillen geblieben sind, so ist es nötig, das Tier zu töten. Erfolgt die Sektion 24 bis 48 Stunden nach der Infektion, so erscheint die Bauchhöhle zunächst nicht verändert. Die Serosa des Darmes und das Peritoneum parietale ist spiegelnd, glatt und glänzend, nur das Netz liegt zusammengerollt unterhalb des Magens, ist ödematös, stärker wie normal gerötet, manchmal mit weiBlichen Fibrinflocken bedeckt und verklebt. Die Lymphknötchen, die sonst nicht sichtbar sind, sind jetzt stark vergrößert, von gelbweißem Aussehen, gleich als wenn sie eitrig erweicht wären. Durch ihre GröBe und gelbweiße Farbe treten sie deutlich hervor (vgl. Taf. II, Zeichnung Nr. 3). Zerquetseht man ein größeres Lymphknötchen und färbt den Ausstrich auf Tuberkelbazillen, so ist man erstaunt über die große Anzahl der sich hier vorfindenden Stäbchen.

Über die Zahl und Lokalisation der Bazillen in den Lymphknoten und dem übrigen Gewebe des Netzes geben Schnittpräparate genauen Aufschluß. Die Menge der Stäbchen innerhalb der größeren Lymphknoten ist oft enorm; färbt man nach Ziehl, so erscheint das Gewebe des Knötchens oft ganz rot. Die Stäbchen liegen teils vereinzelt oder zu 
mehreren in den dicht aneinander liegenden polynukleären Leukozyten, teils finden sie sich angehäuft in einzelnen Zellen, die über das ganze Gesichtsfeld verstreut sind. Außer diesen in Zellen eingeschlossenen Stäbchen sind aber noch große Bazillenrasen vorhanden, die offenbar kleine, trotz sorgfältiger Verreibung nicht in einzelne Stäbchen aufgelöste Bröckel der injizierten Kultur darstellen. Eine Reaktion seitens des Gewebes ist trotz der großen Menge der auf engem Raum zusammenliegenden Bazillen kaum vorhanden. Aber nicht nur die Lymphknoten, sondern auch die kleinen, aus Lymphozyten bestehenden Zellaggregate, sowie die einzelnen oder zu mehreren in dem Maschengewebe des Netzes liegenden leukozytären Elemente enthalten die nach Ziehl rot gefärbten Stäbchen.

Der Weg, den die Bazillen genommen haken, um aus der freien Bauchhöble in das Netz zu gelangen, ist nicht schwer zu verfolgen.

Von einer Vernichtung der Keime durch die eingewanderten Mikround Makrophagen im Peritonealraum selbst kann keine Rede sein, aber als Transportzellen haben die Phagozyten eine groBartige Tätigkeit entfaltet, indem sie fast alle in die freie Peritonealfüssigkeit injizierten Bazillen bis auf wenige Reste, die in Leukozyten eingeschlossen, in der Bauchböhle verblieben sind, in das Netz und zwar hauptsächlich in die Lymphknötchen befördert und dort abgelagert haben (vgl. Taf. III, Mikrophotographie Nr. 1).

Was den weiteren Verlauf der peritonealen tuberkulösen Infektion des Meersehweinchens und Kaninchens anbetrifft, so gehen die durch das Netz abgefangenen Bazillen hier nicht zugrunde, sondern verursachen typische Netzveränderungen. Nach etwa 14 Tagen ist aus dem aufgerollten Netz ein wurstförmiger Tumor von gelbweiBer Farbe und derber Beschaffenheit geworden, der unterhalb der großen Kurvatur des Magens gelegen ist und an Stellen, wo zahlreiche Bazillen hingerieten, bereits verkäst ist. Die Milz ist um diese Zeit auch schon vergröBert, zeigt aber nur feinste, eben sichtbare Knötchen in ihrem Parenchym. Makroskopisch sieht man an den übrigen Organen der Bauchhöhle sowie an den Lungen noch keine Veränderungen. Die miliaren Knötchen der Milz verdanken ihre Entstehung Tuberkelbazillen, die von Phagozyten verschleppt, durch die dem Peritoneum entstammenden Lymphgefäße in den Hauptlymphstrom und sekundär ins Blut eingeschwemmt sind, genau, wie ich es bei den Tuschezellen, die man in der Milz und Leber nach intraperitonealer Injektion von Tusehe antrifft, geschildert habe.

Wir sehen also, daß der Mechanismus der peritonealen Infeition mit Tuberkelbazillen d. h. was die erste Phase, die Resorption der Bazillen aus der Bauchhöhle anbetrifft, genau in derselben Weise verläuft wie beim Tuscheversuch. Sobald die Bazillen innerhalb des lymphoiden Gewebes 
Über dIE Bededtung o. TÄtigkett Des grossen Netzes dsw. 431

des Netzes sich befinden, können sie ihre spezifischen Eigenschaften entfalten, die schlieBlich zur Zerstörung der Phagozyten und Verkäsung des umliegenden Gewebes führen.

Weitere Versuche, den Mechanismus der peritonealen Infektion bei den gewöhnlichen Eitererregern zu erforschen, habe ich bei jungen Kaninchen und Meerschweinchen mit. Staphylo- und Streptokokken angestellt. Om die Tätigkeit des Netzes bei diesen Infektionen frei von Nebenerscheinungen zu beobachten, muß man natürlich untertödliche Dosen nehmen. Beim Staphylococcus, jungen Kaninchen intraperitoneal injiziert, genügt eine Ôse virulenter Agarkultur. Es würde mich zu weit führen, hier den Gang der Infektion genauer zu schildern. Soweit die erste Phase, das Verschwinden der Kokken aus dem Peritonealraum in Betracht kommt, ist er genau derselbe wie bei der Infektion mit Tuberkelbazillen. Die Phagozytose ist bei der Staphylo- und Streptomykose; sofern keine zu großen Mengen verwandt werden, besonders ausgesprochen, gleichgültig, ob man virulente oder wenig virulente Stämme benutzt.

Tötet man ein Staphylo- oder Streptokokkentier nach 24 Stunden, so erscheint das Netz wenig verändert. Es hat sich aufgerollt und nur dort, wo gröBere Lymphknötchen im Netz liegen, sieht man stärkere Rötung und Injektion der GefäBe. Die gröBten, an der Wurzel des Netzes liegenden Lymphknötchen beherbergen große Mengen von in Phagozyten eingeschlossenen Kokken, wovon man sich leicht an nach Gram gefärbten Schnittprāparaten überzeugen kann. Bei dieser Anhäufung von Kokken handelt es sich keineswegs um eine Vermehrung, sondern lediglich um eine Verschleppung und Ablagerung auf dem verhältnismäBig kleinen Raum eines Lymphknötchens durch die Leukozyten. Nirgendwo sieht man, daB die Kokken in das benachbarte Gewebe eindringen, nirgendwo die Zeichen einer progredienten Entzündung.

Die Versuche mit Pneumokokken, Bacterium coli, Diphtherie- und Milzbrandbazillen fielen in derselben Weise aus.

Wie gestaltet sich nun das weitere Schicksal der Bakterien nach ihrer Ablagerung durch die Leukozyten im lymphoiden Gewebe des Netzes?

Nach Metschnikoff, der den Phagozyten die ausschlaggebendf Rolle bei der Vernichtung der Mikroorganismen zuerteilt, sollen bekanntlich die Bakterien im Protoplasma der Lenkozyten verdaut werden und zwar innerhalb der Bauchhöhle.

Es kann jedoch darüber kein Zweifel bestehen, daB eine Reihe von pathogenen Keimen wie z. B. Strepto- und Pneumokolken, Diphtherie- und Typhusbazillen, Choleravibrionen, Bacterium pyocyaneum u.a. zum geringen Teil schon in der freien Peritonealfüssigkeit beim nicht immunisierten 
Tier ihren Untergang finden. Dafür spricht das Vorkommen von Degenerationsformen, die man bei sorgfältiger Untersuchung des Peritonealexsudates stets konstatieren kann. Der gröBere Teil wird allerdings von den Phagozyten aufgenommen. Es fragt sich aber, ob die innerhalb der Leukozyten liegenden Keime hier abgetötet werden oder nicht. Für manche Bakterien, wie z. B. den Tuberkelbacillus, Staphylokokken kann diese Frage heute wohl verneint werden. Bei einer weiteren Reihe ron Keimen, virulenten Strepto- und Pneumokokken, sowie Diphtheriebazillen, babe ich öfter das Peritonealexsudat der infizierten Tiere 24 Stunden nach der Injektion, als ich mich überzeugt hatte, dab sämtliche Keime sich inuerhalb der Phagozyten befanden, auf passenden Nährböden ausgestrichen, aber stets ein gutes Wachstum der betreffenden Bakterien erhalten, ein Beweis, daB diese Keime noch nicht ron den Phagozyten vernichtet waren. Man könnte ja den Einwand machen, daß sich auch noch freie Bakterien im Peritonealexsudat befunden haben könnten, die meiner Kontrolle entgangen wären. Um diesem Einwand zu begegnen, habe ich nicht die freien, noch in der Bauchhọhle befindlichen, sondern die bereits in die Lymphknötchen des Netzes eingewanderten, mit Bakterien beladenen Leukozyten zur Aussaat. auf Nährböden benutzt, indem ich die gröbten Knötchen nach 24 bis 48 Stunden sorgfältig aus der Umgebung herauspräparierte, sie mehreremal in Kochsalzlösung wusch, sie dann zerquetschte und dieses Material auf die Nährböden brachte. Auch hier erbielt ich bei den genannten pathogenen Keimen stets ein reichliches Wachstum. Also selbst im lymphoiden Gewebe des Netzes waren in den ersten Tagen die Keime noch lebensfähig. Die Diphtherietiere starben gewōhnlich nach 3 Tagen. Außer im Netz fanden sich im Organismus des Meerschweinchens keine Bazillen. Der Tod der Tiere ist offenbar auf eine Toxinwirkung von den im Netz abgelagerten Bazillen zurückzuführen.

Versuchstiere, die mit Strepto-, Staphylo- und Pneumokokken infiziert worden waren, blieben dagegen am Leben. Hier muBte also allmählich eine Abtötung innerhalb der Lymphknötchen des Netzes erfolgt sein. Wenn man bedenkt, eine wie große Menge der pathogenen Keime in den gröBten dieser Lymphknötchen zusammengetragen wird, so läBt sich daraus der SchluB ziehen, daB die bakterizide Kraft eines derartigen Zellkomplexes eine auBerordentliche sein muB. Wie die Bazillen hier vernichtet werden, kann ich allerdings nicht sagen. Der Vorgang des Unterganges von Bakterien ist uns ja auch bei den Lymphdrüsen des übrigen Körpers nicht bekannt, nur soviel wissen wir, daB ihr bakterizides Vermögen ein gewaltiges ist.

Gegenüber diesen Versuchen am nicht präparierten Tier war es ron grobem Interesse, die Vorgānge in der Bauchböhle immunisierter Tiere zu verfolgen. 
Über die Bedeutung v. Tätigkeit des Grossen Netzes usw. 433

Wenn man Meerschweinchen und Kaninchen mit Strepto-, Pnéumokokken, Milzbrandbazillen u. a. vorbehandelt, so gewinnt das Peritonealexsudat dadurch eine außerordentlich starke bakterioly tische Fähigkeit für diese Bakterien, eine Tatsache, die uns durch die klassischen Untersuchungen R. Pfeiffers für andere Bakterien, den Choleravibrio und Typhusbacillus bekannt ist. Durch diese starke bakterizide Tätigkeit verschwinden die Baliterien sehr schnell aus der Bauchhöhle, indem sie in der Peritonealflüssigkeit zum gröBten Teile aufgelöst werden. Wichtig ist, daB im immunisierten Tier eine Reaktion von seiten der aus dem Blut stammenden polynukleären Leukozyten fast gänzlich ausbleibt, während Makrophagen gewöhnlich in großer Anzahl im Exsudat, vielfach zu kleinen Häufchen verklumpt, 'aus dem Netz in die freie Bauchhöhle eingewandert sind. Die Auflösung der Balkterien lăßt sich besonders gut an sporenhaltigen Milzbrandbazillen studieren. MIan kann unter dem Mikroskop verfolgen, wie die Konturen der Bazillen allmählich verschwinden, während die einzelnen Sporen des Stäbchens keine Veränderungen erleiden. Der ProzeB verläuft sehr schnell, so daB man 20 Minuten nach der Finspritzung nur Sporen, die allerdings ebenso wie ein.Teil der Bazillen gierig von den Makrophagen gefressen werden, im Exsudat finden kann. In derselben Weise verläuft der ProzeB der Auflösung bei den Strepto- und Pneumokokken, gleichzeitig allerdings auch hier mit einer energischen Phagozytose seitens der Makrophagen, die mit den Resten und Trümmern der Balterien rom Netz aufgenommen werden. Ein anderer Teil wird direkt von den Lymphbahnen des Lymphnetzes resorbiert.

Die Darstellung, die ich von den beim Cntergang der Bakterien in der Bauchhöhle sich abspielenden Vorgängen gegeben habe, weicht in einer Reihe von Punlten von der zurzeit herrschenden Lebre ab. Vor allem kann ich der Ansicht Metschnikoffs, dab die Vernichtung der Keime im Peritonealraum durch die eingewanderten Phagozyten vor sich gehe, nicht zustimmen.

Der ProzeB beim vorbehandelten ist wesentlich verschieden von dem beim normalen Versuchstiere. Während beim immunisierten Tier der Untergang der Keime in der Hauptsache durch die bakteriolytische Tätigkeit des Peritonealexsudats, also extrazellulär im Sinne R. Pfeiffers schnell entschieden wird, ist der Verlauf beim nicht immunisierten Versuchstier viel langsamer und komplizierter, indem die Vernichtung der Keime hauptsächlich im lymphoiden Gewebe des Netzes vor sich geht, wohin die Bakterien teils direkt resorbiert, teils indirekt durch die Mikro- und Makrophagen abgelagert werden. 
Das Schicksal der in die Bauchhöhle eingeführten pathogenen Keime wird also nicht durch einen einzigen, sondern durch eine Reihe von mitwirkenden Faktoren entschieden. Wenn wir sie hinsichtlich ihrer Beteiligung an der Keimabtötung prüfen, so können wir sagen, dab die Phagozytose dasjenige Phänomen ist, das bei sämtlichen Bakterien regelmäBig und am sinnfälligsten in die Erscheinung tritt, während der Vorgang der Auflösung im Exsudat sich meist dem Auge des Beobachters entzieht. $\mathrm{DaB}$ die Phagozyten virulente, lebensfähige Keime aufnehmen können, muß zugegeben werden. Keineswegs ist aber die Aufnahme gleichbedeutend mit einer Abtötung im Leukozytenleibe, vielmehr tritt diese erst innerhalb der einzelnen Lymphknötchen und Drüsen ein. In diese mit hoher bakterizider-Kraft versehenen Stätten gelangen sie allerdings durch die Wanderzellen. Die Tätigkeit der letzteren ist also dahin zu charakterisieren, $\mathrm{daB}$ sie in erster Linie Resorptionszellen sind, wie eine Reihe hervorragender Forscher, z. B. R. Pfeiffer, Baumgarten u. a. stets hervorgehoben haben. 
Über die Bedeutung u. Tätigkeit Des grossen Netzes osw. 435

\section{Erklärung der Abbildungen. \\ (Taf. II u. III.)}

\section{Tafel II.}

Zeichnung Nr. 1 u. 2.

Stellt einen Schnitt durch den proximalen Teil des großen Netzes eines jungen Kaninchens $\left(1500^{\mathrm{grm}}\right.$ ) bei schwächerer VergröBerung (Winkel Oc. 3 Obj. 3 ) dar. Man sieht in dem Maschenwerk des Netzes ein gröBeres, aus Lymphozyten bestehendes Lymphknötchen and zwei kleinere, ebenfalls aus Lymphozyten bestehende Zellaggregate. Die überall in Gewebe sichtbaren eosinophilen Zellen sind in der Figur Nr. 2 bei starker Vergrößerung (Zeis s Apochrom. Comp. Oc. 8) dargestellt.

\section{Zeichnung Nr. 3.}

Ist angefertigt rom Netz eines $1500 \mathrm{grm}$ schweren jungen Kaninchens (natürliche GröBe), das eine Tuberkelbazillenemulsion $(0.03 \mathrm{grm})$ intraperitoneal erhalten hatte. Auf der Zeichnung treten die gelbweißen kleineren und größeren Lymphknötchen, die besonders im Verlauf der GefäBe sich finden, plastisch hervor. Das 'lier war 48 Stunden nach der Einspritzung getötet worden. Unten links am Netz liegt die Milz.

\section{Tafel IIT:}

Mikrophotogramm Nr. 1. (Vergr. 50 fach.)

Zeigt bei schwacher Vergrößerung einen Schnitt eines großen Lymphknötchens des vorhergehenden Bildes. Die schwarzen unregelmäBigen Schollen, die sich besonders an den Rändern des Drüsengewebes finden, stellen Bröckel der Bazillenemulsion dar, die als solche von Lenkozyten in das Lymphlinötchen transportiert wurden. Die große längliche schwarze Stelle oben rechts ist ein Kunstprodukt (Umschlagsfalte im Sehnitt).

Mikrophotogramm Nr. 2. (Vergr. 900 fach.)

Auf dem Bild ist ein LymphgefäB vom groBen Netz zu sehen, in dessen Inneren sich zahlreiche, meist degenerierte Wanderzellen befinden, die mit Milzbrandbazillen oder deren Resten erfüllt sind. Es finden sich aber auch freie Trümmer von Milzbrandbazillen innerhalb des Lymphgefäßes. Das Tier war mit einer Öse virulenter Milzbrandkultur intraperitoneal geimpft und nach etwa 30 Stunden verendet.

' Die Mikrophotogramme verdanke ich der Güte des Hrn. Prof. Zettnow. 

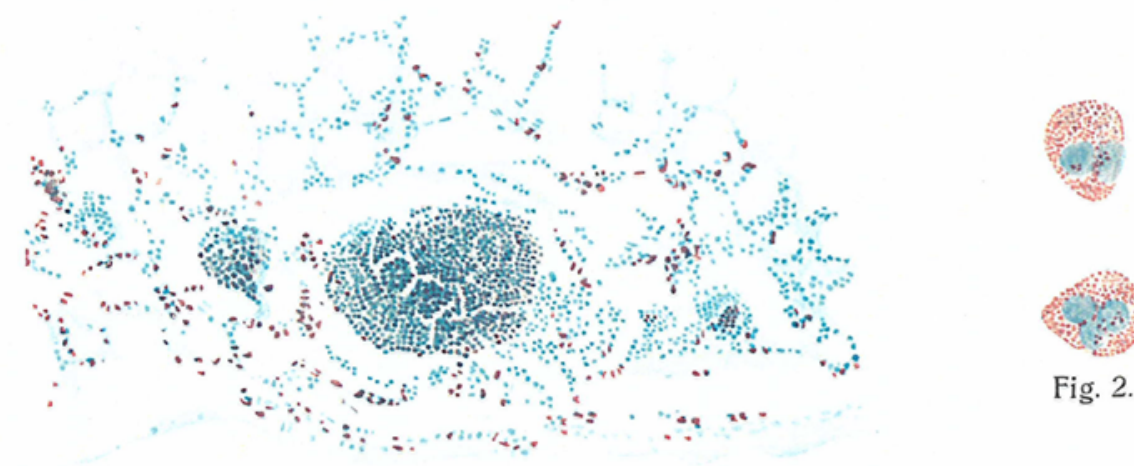

Fig. 1.

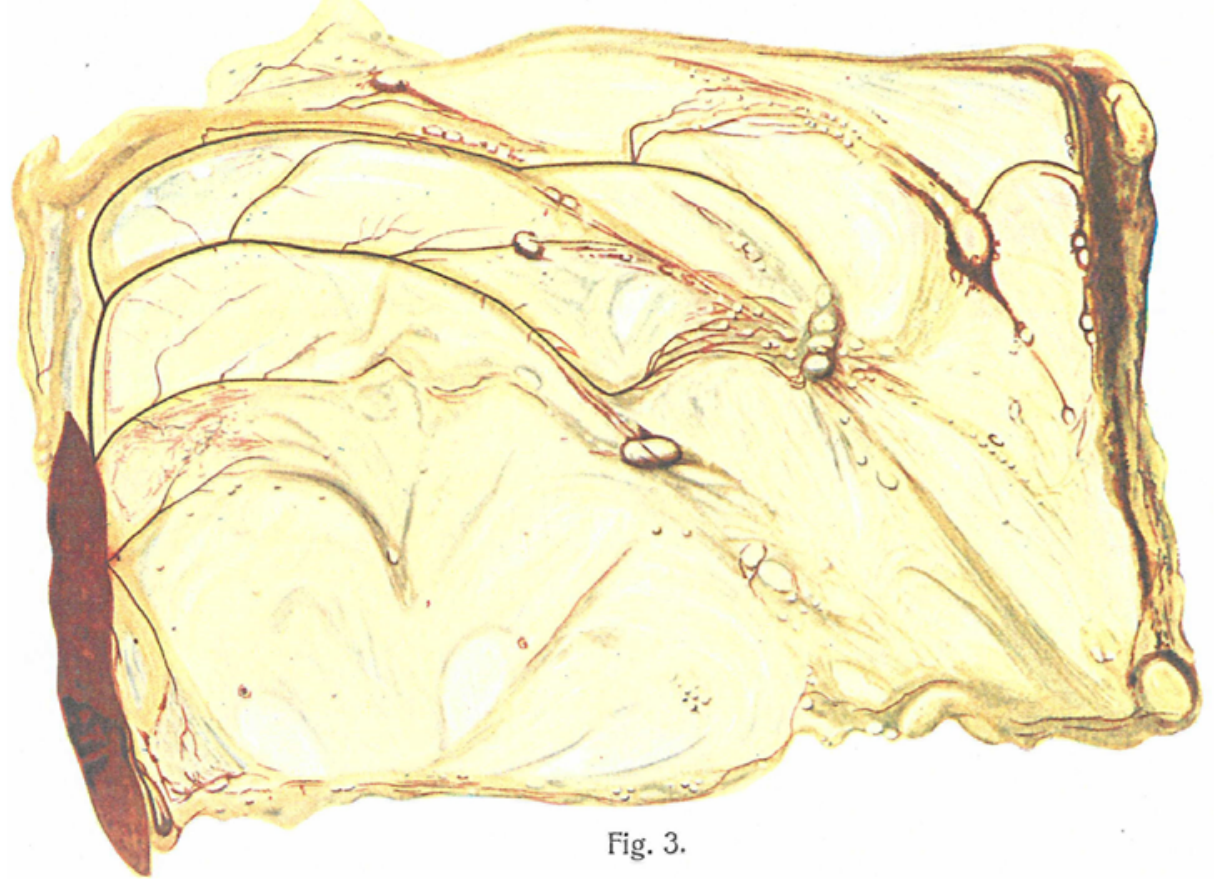

Verlag von VEIT \& COMP., Leipzig. 


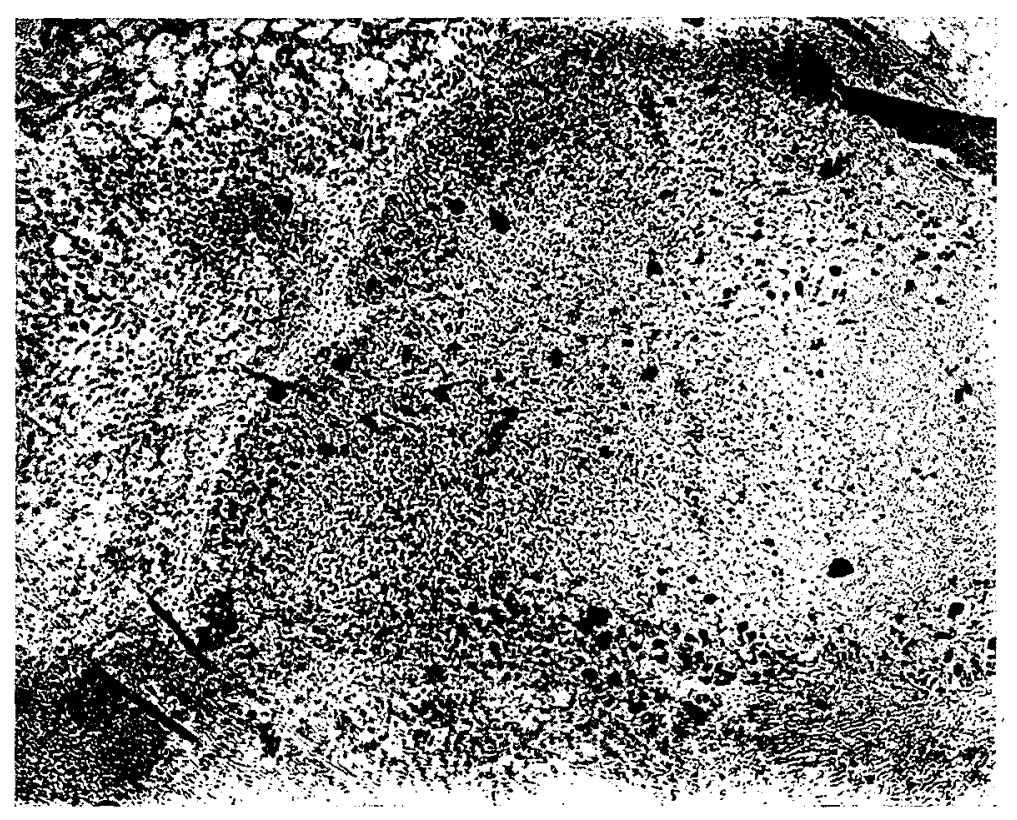

Mikrophotogramm Nr. 1.

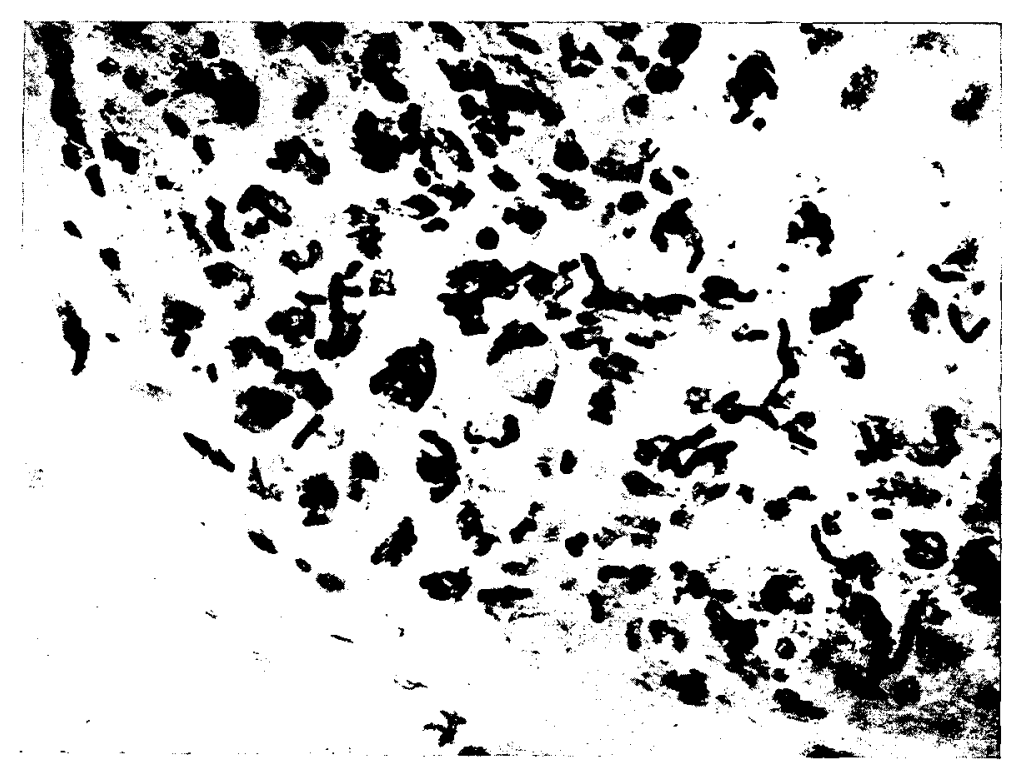

Mikrophotogramm Ni. 2. 